\title{
TEORÍAS IMPLÍCITAS SOBRE LA ESTABILIDAD DE LA NATURALEZA HUMANA Y DEL ENTORNO SOCIAL, Y SU RELACIÓN CON LA REINCIDENCIA DELICTIVA EN INTERNOS RECLUIDOS EN EL CENTRO DE CUMPLIMIENTO PENITENCIARIO DE LA COMUNA DE PUNTA ARENAS ${ }^{1}$
}

\author{
ERIC MOLINET OYARZÚN*, DANIEL VELÁSQUEZ CASTRO* Y CLAUDIA ESTRADA GOIC**
}

\begin{abstract}
RESUMEN
El presente informe de investigación se ocupa de la posible relación entre la reincidencia delictual y las teorías implícitas sobre la estabilidad de la naturaleza humana y del entorno social. Su hipótesis central es que poseer una visión estática sobre la naturaleza humana y del entorno social se acompaña de mayores tasas de reincidencia. Nos basamos en los postulados de Dweck (1996) que indican que aquellos que poseen creencias respecto a que las personas poseen un núcleo central relativamente fijo y no maleable, tienen expectativas respecto a que las personas no cambian y se comportan en consonancia con esta visión. En un estudio en el que participaron 48 reos de la ciudad de Punta Arenas, se observó la existencia de una relación significativa entre ambas variables. Las altas reincidencias se acompañaron de una visión estática de la naturaleza humana y del entorno social mientras que las bajas reincidencias se asociaron a visiones más flexibles. Las implicancias de este resultado para la intervención psicosocial en reinserción son presentadas junto a las nuevas preguntas que genera esta investigación preliminar.
\end{abstract}

PALABRAS CLAVES: Teoría implícita, reincidencia, identidad social.

\section{RELATION BETWEEN IMPLICIT ABOUT STABILITY OF HUMAN NATURE, SOCIAL ENVIROMENT AND CRIMINAL RECIDIVSM IN PRISIONERS FROM PUNTA ARENAS PENITENCIARY}

\footnotetext{
ABSTRACT

This investigation report deals with a possible relation between tortious recidivism and the implicit theories about stability of the human nature and social environment. Its main hypothesis is that having a static view of the human nature and social environment implies higher recidivism rates. We are based on Dweck postulates (1996) which state that those having beliefs regarding that people have a relatively fixed and not malleable central core, think that people do not change, so they behave in relation to this view. In a study carried out in Punta Arenas in

$1 \quad$ Esta investigación fue financiada por el Proyecto Fondecyt 11060001.

- Facultad de Humanidades, Ciencias Sociales y Ciencias de la Salud. Departamento de Ciencias Sociales. Trabajo Social. Universidad de Magallanes. Punta Arenas - Chile.

" Facultad de Humanidades, Ciencias Sociales y Ciencias de la Salud. Departamento de Ciencias Sociales. Psicología. Universidad de Magallanes. Punta Arenas - Chile.

Correspondencia: Claudia Estrada Goic: Psicología. Universidad de Magallanes. Av. Bulnes 01855, Punta Arenas. E-mail: claudia.estrada@umag.cl
} 
which 48 prisoners took part, a significant relation between both variables was found. High recidivisms were linked to a static view of the human nature and social environment whereas low recidivisms were linked to more flexible views. Implications of this result for the psychosocial intervention for reintegration are presented together with the new questions arisen from this preliminary investigation.

KEY WORDS: Implicit theory, recidivism, social identity.

\section{INTRODUCCIÓN}

Sin importar en qué contexto social o cultural nos encontremos, la delincuencia es un tema de importancia transversal debido al impacto que este fenómeno tiene en la convivencia y el bienestar social. Desde que el hombre es hombre el comportamiento antinormativo a resultado intrigante para las ciencias sociales y en particular para la psicología social, disciplina desde la que han surgido numerosos modelos explicativos del mismo. Uno de los aspectos más interesantes de esta problemática es la reincidencia delictual o delictiva, es decir, qué lleva a una persona que ha sido detenida por un delito y que ha debido cumplir una condena, a volver a cometer actos similares (Código Penal, 2002, art. 494 bis, inciso 4) La reincidencia delictiva se ha mostrado como un problema complejo que requiere del abordaje de una multitud de factores explicativos dentro de los cuales podemos mencionar la disfuncionalidad familiar, la deserción escolar, la cesantía y la existencia de subculturas. La gran cantidad de información recogida en estudios sobre esta temática ha permitido el desarrollo de programas interdisciplinarios orientados al logro de lo que ha sido llamado "la reinserción social" o la "rehabilitación". Aunque existe acuerdo que ni en nuestro país ni en nuestra región existen condiciones ideales para el desarrollo de estos programas, gran parte de ellos aspira a mejorar la integración familiar, el acercamiento a las redes primarias y secundarias, la nivelación escolar y la capacitación, como formas de intervención en vistas a disminuir la reincidencia luego del cumplimiento de la condena. A pesar de los esfuerzos humanos y materiales desplegados con el fin ya señalado, la eficacia de estos programas ha resultado ser insuficiente ya que se calcula que este fenómeno presenta tasas nacionales y regionales cercanas al $60 \%$ con una leve pero consistente tendencia al aumento. Así es que nos encontramos frente a la paradoja de que hoy más que nunca antes estamos ante un fenómeno que ha sido de interés científico y que se ha traducido en diversos programas de intervención, sin que estemos por ello, logrando niveles de éxito en el enfrentamiento de esta problemática. Nuestro aporte consiste en una mirada alternativa de este problema que considera un nuevo factor asociado que no ha sido considerado hasta ahora y que podría contribuir al entendimiento y enfrentamiento del mismo.

\section{La reincidencia delictual, un fenómeno complejo}

La delincuencia no es un fenómeno unidimensional y existen diversos desarrollos teóricos para explicarla. Un amplio conjunto de teorías se pronuncian frente a este fenómeno desde disciplinas complementarias tales como la sociología, la antropología, la psicología social o criminalística, entre otras. Una de las visiones más universalmente aceptadas es la propuesta por la criminóloga chilena Doris Cooper (2007) quien señala que el modelo económico es la causa principal de la delincuencia. Su postura se basa en numerosos estudios realizados en nuestro país que indican la existencia de una asociación entre la delincuencia y la pobreza. Sin embargo, siendo el modelo económico un factor explicativo de peso, no se ha convertido en la respuesta principal a este problema ya que no revela el por qué en las sociedades consideradas desarrolladas también se observa un aumento sostenido de la delincuencia y tazas de reincidencia similares a las observadas en nuestra región (Muñoz, 1996).

Respecto de las causas que se asocian a la reincidencia delictiva y que también han sido mencionadas como causas de la delincuencia, tenemos:

a) La exclusión social que sufren aquellos que tienen antecedentes delictuales. Aunque hayan recibido capacitación durante el cumplimiento de su condena, al volver enfrentan cesantía y marginación 
prolongada lo que no sólo impide la reinserción sino que potencia el surgimiento de sentimientos de frustración que favorecen la aparición de comportamientos violentos y otros delitos asociados.

b) La baja competitividad debida al abandono escolar temprano que determinan dificultades importantes al momento de buscar una inserción laboral digna.

c) Un ambiente familiar disfuncional, muchas veces violento, que se acompaña de pautas de interrelación inadecuadas dificultando el desarrollo de habilidades sociales asociadas a valores sociales de convivencia, comunicación, formas de resolver y enfrentar los conflictos y baja tolerancia a la frustración. Cuando una persona abandona el centro penitenciario vuelve a los patrones conocidos de comportamiento familiar.

d) Ruptura del vínculo social, redes sociales más frágiles en la que las personas no pueden apoyarse con eficacia, o por el contrario, un vínculo social fuerte asociado a una subcultura delincuente que castiga los intentos de aculturación de sus miembros.

e) La cultura de la violencia y del consumismo: Reflejada en la televisión, juegos y deportes.

Los factores anteriores se encuentran, además, con algunas causas institucionales que estimulan la conducta delictiva y que se asocian a la inadecuación o ineficacia del sistema de justicia penal y los pobres instrumentos de rehabilitación con que cuenta el medio cerrado (Cooper, 1994)

\section{Reincidencia e identidad social}

Como fue introducido anteriormente, el problema de la reincidencia es relevante tanto por su significación social como por su magnitud. A pesar de los esfuerzos desarrollados en lo que han sido llamados "programas de rehabilitación", las tazas de reincidencia aumentan en el tiempo. El mensaje parece ser que romper con el ciclo delictivo requiere más que una intervención social que apunte a las causas conocidas de la delincuencia y que parece ser el momento de apoyar a este proceso a través de la comprensión del fenómeno desde una perspectiva identitaria. Según Cooper (2007), el 72\% de los niños privados de libertad se percibe a sí mismo como un ladrón verdadero. Esto no sólo significa que se proyectan hacia el futuro a partir de esta autodefinición sino que su identidad social se encuentra determinada por la pertenencia a este grupo social. La presencia de códigos y roles que definen al tipo de delincuente más prestigioso otorga las pautas que el grupo de pertenencia posee para aumentar la positividad de cada uno de sus miembros en las que un niño que busca mejorar su autoconcepto social buscará ser un asaltante más que un lanza, este último uno de los menos valorados dentro de esta subcultura. Según la misma autora, un $62 \%$ de los condenados urbanos masculinos se reconoce como parte del Hampa y se autocategorizan como ladrones - ladrones determinando asi aspectos psicosociales como valores, motivaciones, actitudes, percepciones, cogniciones, comportamientos físicos, etc. Desde la psicología social, la teoría de la identidad social (Tajfel 1972, 1981) plantea que las personas construyen una parte importante de su visión de sí mismo a partir de sus pertenencias a grupos sociales. Este aspecto del sí mismo, denominado identidad social, no sólo es un elemento fundamental de lo que cada uno es sino también el punto de partida del proceso de comparación social a partir del cual conocemos que tan deseables somos socialmente. Esta teoría establece que las personas buscan pertenecer a grupos que son valorados positivamente de forma de aportar a su auto-concepto. Cuando un individuo es miembro de un grupo con una identidad social negativa tiene la posibilidad de enfrentar este problema movilizándose para cambiar de un grupo a otro más positivo. En el caso de la pertenencia a la categoría social de delincuentes, el individuo puede emigrar del grupo anti-normativo hacia uno más normativo o socialmente aceptado. Para que esto pueda ocurrir es necesario que la categoría alternativa sea más positiva socialmente que la que posee (¿Resulta mejor dejar de ser delincuente internacional para ser obrero?) y que el individuo perciba que este cambio es posible, lo que técnicamente ha sido denominado "percibir permeabilidad en las fronteras del grupo". En otras palabras, una persona cambia de categoría social si esta es negativa, si la opción es más positiva y si piensa que es posible. Es sobre este último punto que se ocupa la investigación que presentamos, esto es, la asociación que existe entre las creencias de que el cambio individual y social no es posible, y la reincidencia delictual. 


\section{Las Teorías Implícitas:}

Cuando hablamos de teorías implícitas sobre la estabilidad de la naturaleza humana y del entorno social, nos referimos al conjunto de creencias que las personas tienen respecto al potencial de cambio de las personas y del entorno social. Las teorías implícitas tienen una función pragmática ya que son utilizadas para interpretar los sucesos de su entorno y hacer inferencias prácticas que condicionan su acción (Rodrigo, Rodríguez y Marrero, 1993). El concepto de teoría implícita fue acuñado por Bruner y Taiguri (1954) y refiere a un conjunto organizado e implícito de creencias sobre la personalidad. Desarrollos posteriores del concepto han permitido su uso para hacer referencia a la naturaleza humana o de los grupos. Dweck y sus colegas (Dweck, Hong y Chiu, 1993, 1995) se han interesado en las teorías que las personas tienen respecto de ciertos aspectos de la naturaleza humana. Estos autores señalan que es posible agrupar a las personas según dos grandes categorías de teorías implícitas que se aplican a la inteligencia, el carácter moral y la personalidad: los entiteístas y los incrementalistas. Los primeros creen que las características de una persona se organizan en torno a un núcleo central relativamente fijo y no maleable. Numerosas investigaciones han señalado que los teóricos entiteístas, tienden a dar juicios más definitivos, más causales y a partir de informaciones parciales, dicotomizando la información negativa y positiva, establecen juicios diagnósticos a partir de un proceso de inferencia y realizan atribuciones disposicionales. Los teóricos incrementalistas, por su parte, creen que la configuración general de una persona es relativamente maleable y susceptible de un desarrollo continuo. En general, tienden a realizar juicios en términos de procesos mediacionales para comprender el comportamiento de los otros y prestan atención a la influencia de los factores situacionales (Dweck et al, 1995). Los estudios sobre los efectos de las T.I.P. en relación con los niveles personales e interpersonales indican que estas diferentes teorías tienen impacto en la forma en que se explica el rendimiento intelectual, la orientación hacia los "deberes y derechos", la evaluación de la trasgresión y la asignación de castigo y el manejo de la información inconsistente. Respecto de sus efectos sobre el nivel social, los resultados muestran que los teóricos entiteístas adhieren, más a los estereotipos cuando estos parecen reflejar diferencias innatas entre los grupos, son más extremos en sus apreciaciones y prefieren la información estereotípica. Los trabajos realizados por Dweck (1996) indican que las teorías implícitas son manipulables y por lo tanto susceptibles de cambiar aunque tienden a ser autoconfirmatorias lo que dificulta su modificabilidad.

A partir de este desarrollo teórico, nos preguntamos si las personas reincidentes tienen teorías implícitas diferentes sobre la posibilidad del cambio de las personas y del entorno social. Ya que las teorías implícitas actúan como ideas preconcebidas que afectan nuestra visión de mundo, serían determinantes al momento de percibir la posibilidad o no de cambiar. Si una persona piensa que las personas no son susceptibles de cambiar profundamente y piensan que el entorno social tiende al status quo, esta creencia también tendrá repercusiones en la construcción de su identidad social. Si pienso que los otros no cambian tendré una visión estática de la realidad que me incluye a mi mismo. Es por esto que planteamos la posibilidad de que exista una asociación entre los niveles de reincidencia y la tendencia a poseer una visión entiteísta de la realidad. Creemos que aunque la existencia de esta asociación no nos permitirá establecer una relación causa efecto entre ellas, si conduce a introducir una nueva variable explicativa a la problemática de la reincidencia que no se sitúa únicamente en el espacio social sino en el la interacción entre las creencias individuales y lo social.

\section{OBJETIVO.}

La investigación tuvo como objetivo interno central determinar la existencia o no de una relación entre la concepción que los delincuentes poseen sobre la naturaleza humana y social, y el número de reincidencias.

\section{HIPÓTESIS.}

HI: Existe una relación entre las Teorías Implícitas sobre la naturaleza humana, y sobre el entorno social y la reincidencia delictiva

HO: No existe una relación entre las Teorías Implícitas sobre la naturaleza humana y sobre el entorno 
social y la reincidencia delictiva

HI: $r x y \neq 0$.

$\mathrm{HO}: \mathrm{rxy}=0$.

$\mathrm{x}$ : Reincidencia.

y: TI sobre naturaleza humana y entorno social.

\section{MÉTODO}

Participantes. La muestra no probabilística de tipo intencional estuvo conformada por 48 personas recluidas en el Centro de Cumplimiento Penitenciario de la comuna de Punta Arenas quienes participaron voluntariamente en el estudio.

Instrumentos: Para medir las teorías implícitas sobre la naturaleza humana y el entorno social se utilizó un cuestionario compuesto de 13 afirmaciones adaptadas de Dweck (1996) que debían ser contestadas sobre escalas tipo Likert de 4 puntos (para evitar el punto neutro) que fueron desde 1 (totalmente en desacuerdo) hasta 4 (totalmente de acuerdo). Estas afirmaciones miden el grado en que las personas están de acuerdo o no con la idea de que la naturaleza de las personas es estable y no cambia en el tiempo (10 afirmaciones) y con la idea de que el entorno social tiende a mantenerse igual sin experimentar cambios importantes (3 afirmaciones). Puntuaciones altas indican una visión estática de la naturaleza individual y social. La confiabilidad de este cuestionario alcanzó niveles aceptables (alfa de Cronbach: 0,67).

El número de reincidencias fue obtenido a partir de la información contenida en los registros disponibles en el centro penitenciario.

Procedimiento. Los participantes fueron contactados uno a uno en el centro penitenciario e invitados a responder al cuestionario y a permitir el acceso a la información sobre su reincidencia. Cada uno recibió información respecto a la confidencialidad de la información individualizante y los objetivos generales del estudio. Al finalizar la aplicación se contestó a sus preguntas y se les agradeció por su participación.

\section{RESULTADOS}

La muestra mostró un promedio de edad de 34 años, con un nivel de escolaridad media dentro del rango "básica completa".

El puntaje promedio alcanzado en el cuestionario fue de 34, 20 (de un mínimo posible de 13 puntos y un máximo de 52).

El promedio de reincidencias fue de 2,7. Un 21,3\% mostró baja reincidencia (1), un 47,9\% reincidencia media ( 2 y 3 ) y un $20,8 \%$ alta (más de 4 reincidencias).

El análisis de correlación muestra que existe una asociación positiva entre los puntajes en el cuestionario de teorías implícitas sobre la naturaleza humana y el entorno social, y la cantidad de reincidencias. En otros términos, tal como fue hipotetizado, las altas reincidencias se asocian a una teoría implícita estática de la naturaleza humana y social mientras que la baja reincidencia se relaciona con una teoría implícita dinámica, $r_{(48)}=0,34, p<0,02$

Al comparar los tres rangos de reincidencia (baja, media y alta) según el puntaje obtenido en el cuestionario de teorías implícitas, se observa que los grupos son significativamente diferentes, $F_{(2,45)}$ $=4.77, p<0,013$. Las personas que presentan altos niveles de reincidencia muestran un puntaje promedio mayor $(M=39,1, D T=4,33)$ que las personas con niveles de reincidencia mediana $(M$ $=32,2, D T=6,51)$ y baja $(M=34,0, D T=5,72)$. Los niveles altos de reincidencia presentan puntajes que se acercan significativamente más al polo de las teorías implícitas estáticas respecto de la naturaleza humana y social que los niveles bajos de reincidencia (ver figura 1).

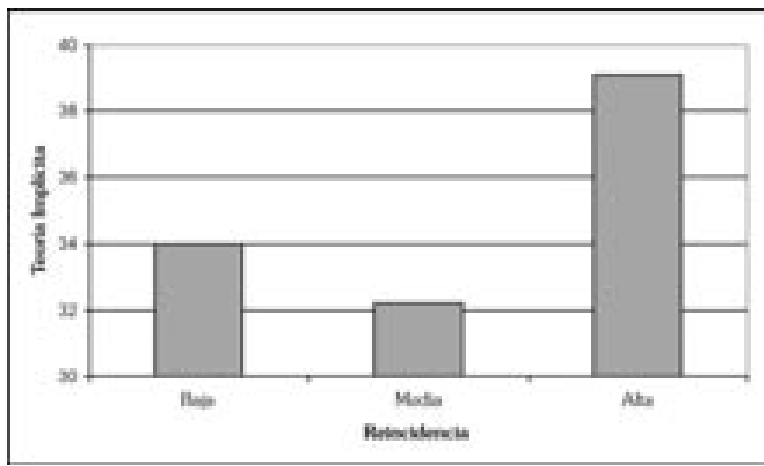

Fig. 1. Puntaje promedio en cuestionario de T.I. según rango de reincidencia 


\section{DISCUSIÓN Y CONCLUSIONES}

El primer resultado que resulta interesante, es el de la distribución de la reincidencia delictiva, definida como el reingreso al sistema carcelario, o el reencarcelamiento. Observamos que dentro de la muestra utilizada para la presente investigación existe un bajo porcentaje de personas que se ubican dentro del rango de Alta Reincidencia (20,8\%). Esto se puede explicar ya que el mayor porcentaje de internos que componen la muestra del estudio, pertenece a una población etárea joven o adultojoven, $y$ debido a esto no suman un número alto de reincidencias. Otra explicación a este fenómeno es que es posible que los internos de esta muestra se encuentren cumpliendo condenas más altas, debido a delitos más importantes, situación que les impiden reincidir.

En concordancia con nuestras hipótesis, los resultados muestran una asociación positiva entre al visión estática de la naturaleza humana y social, y la reincidencia delictual. Mientras más alta es el número de reincidencias, mayor es la adherencia a la teoría implícita según la cuál ni las personas ni los entornos sociales cambian. Los hallazgos obtenidos mediante la aplicación de la escala de teorías implícitas, indican que en cerca del 12\% de los casos observados un puntaje alto en la escala de la percepción del contexto individual se acompaña de una alta reincidencia delictiva y viceversa. En otros términos, mientras más estática es la visión que un participante tiene de la capacidad de cambio de un individuo y su entorno social, más probable es que muestre altos índices de reincidencia. Por el contrario, mientras más flexible es la visión de cambio individual y social, menor es la reincidencia. Este resultado es compatible con la postura de Dweck (1996) que indica que la teoría que una persona posee respecto de la naturaleza humana puede afectar poderosamente en como actuamos. En estudios realizados por este autor, fue posible observar que aquellos que tienen ideas más estables (o inflexibles) de la realidad tienen menor capacidad para adaptarse a los cambios ya que ven a los otros y así mismo como personas "terminadas" que no evolucionan en su esencia. Desde la mirada de la "Cultura del Hampa" (Cooper, 1994), podemos avanzar la idea de que la construcción de la identidad de una persona se lleva a cabo también en función de la subcultura en la que vive. Por lo tanto, una persona que tiene una identidad antisocial, se ve a sí mismo como parte de este grupo cultural y piensa que las personas no cambian, tiene, evidentemente, mayor probabilidad de reincidir. ¿Es la fuerza de la cultura la que lleva a la persona a verse a sí mismo de manera estable o es la mirada estable de la naturaleza humana la que lleva al individuo a construir una identidad delincuente estable? Es una pregunta abierta a la reflexión.

Aunque no es posible establecer una relación causal entre ambas variables, esta asociación nos lleva a plantearnos el viejo dilema en psicología social de si es la creencia la que determina la conducta o es el comportamiento efectuado el que busca consonancia desarrollando una creencia afín. Si es la reincidencia la que lleva a la persona a entender que su imposibilidad de cambiar es el reflejo de una realidad humana y social estática, o si es este tipo de creencias lo que se encuentra a la base de la resistencia al cambio no es, finalmente, el aporte central de esta investigación inicial. Su verdadera contribución se sitúa en posicionar un nuevo factor contribuyente del proceso, la "relación entre la creencia y la conducta", lo que permite una nueva manera de entender el fenómeno en el que factores socio cognitivos se revelan de interés. Pensamos que los avances en esta línea de investigación, tanto a nivel regional como nacional, bien podrían cambiar la forma de enfocar el proceso de la rehabilitación poniendo atención a las creencias que sostienen la identidad social de los individuos que han cometido actos anti-normativos.

Esto concuerda con lo planteado por algunos autores tales como Leyens (1983), respecto de que las teorías implícitas incluyen las ideas sobre la bondad o la maldad de la raza humana, sobre su maleabilidad, su forma de ser, etc., que una persona tiene con relación a una sociedad determinada y que de acuerdo a esto actúa en dicha sociedad.

Nos pareció pertinente realizar un análisis complementario separando la variable reincidencia en tres grupos equiparables (baja reincidencia, mediana reincidencia y alta reincidencia). El análisis indica que los grupos de baja y mediana reincidencias son significativamente diferentes al grupo que se compone de internos con alta reincidencia. Estos últimos poseen una visión menos maleable de la realidad individual y social que aquellos que 
se encuentran en alguno de los otros dos grupos. No es posible establecer con claridad, si es está visión de la realidad lo que hace que las personas reincidan o es la reincidencia lo que potencia una visión de que las personas y los contextos son los que son y que no cambian. Este resultado puede ser discutido a la luz de la teoría de la "profecía que se auto-cumple" (Rosenthal y Jacobson, 1971) que señala que tendemos a actuar de forma de evocar en los otros o provocar en nosotros mismos, conductas que confirman las expectativas previas. Es decir, esta teoría plantea que si una persona tiene la expectativa de que no cambiará en el tiempo y que su entorno social tampoco lo hará se comportará de forma de confirmar esta creencia, no cambiando, no percibiendo ni estimulando cambios en su entorno. Nos parece que está visión teórica es compatible con nuestros resultados y establecen una posible relación causal que deberá ser probada en futuras investigaciones.

\section{BIBLIOGRAFÍA}

Código de Penal (2002) Santiago: Editorial Jurídica de Chile COOPER, D. (2007) Los políticos están ciegos. www.lanacion.cl

COOPER, D. (1994a) Código ético, estratificación social de los ladrones y el nuevo rol del ladrón internacional. Ponencia presentada al XIII, Congreso Mundial de Sociología, Bielefeid, Alemania,

COOPER, D. (1994b). Delincuencia común en Chile. Santiago: Lom

MUÑOZ, O. (1996) Diagnóstico de la delincuencia en Chile entre 1986 y 1995. En Análisis empírico de la delincuencia en Chile 1986 - 1995. Santiago: Fundación paz Ciudadana.
RODRIGO M.J., RODRÍGUEZ A. y MARRERO J., (1993). Las teorías implícitas. Madrid: Visor.

BRUNER, J. S. y TAGIURI, R. (1954). The perception of people. (Vols. 2) Cambridge: Addison-Wesley.

DWECK, C.S., CHIU, C. y HONG, Y. (1995). Implicit theories and their role in judgments and reactions: A world from two perspectives. Psychological Inquiry. Vol.6, No.4, pp.267-285.

DWECK, C.S. (1996). Capturing the dynamic nature of personality. Journal of Research in Personality. Vol. 30, Iss.3, pp.348-362

DWECK, C. S., HONG, Y., y CHIU, C. (1993). Implicit theories: Individual differences in the likelihood and meaning of dispositional inference. Personality and Social Psychology Bulletin, Vol.19, pp.644-656.

RODRÍGUEZ, F.J. y PAÍNO, S.G. (1994). Violencia y desviación social: bases y análisis para la intervención. Psicothema, Vol. 6, 2, 229-244

ROSENTHAL, R. A., y JACOBSON, L (1971). Pygmalion à l'école. Tournai: Casterman.

TAJFEL H. y TURNER J.C. (1986). The social identity theory of intergroup behaviour. En S. Worchel y W.G. Austin (Eds.) Psychology of intergroup relations. (pp.7-24). Chicago, MI: Nelson-Hall

TAJFEL, H. (1972). La catégorisation sociale. En S. Moscovici (Ed.), Introduction à la psychologie sociale (Vol. 1). Paris : Larousse.

TAJFEL, H. (1981). Human groups and social categories. Cambridge: Cambridge University Press. 
